\title{
EXPERIMENTAL STUDY ON SUPPRESSION OF VORTEX STREET BEHIND PERFORATED SQUARE CYLINDER
}

\author{
Amir Teimourian, Hasan Hacişevki, Arian Bahrami \\ Aerodynamic Laboratory, Department of Mechanical Engineering, Eastern Mediterranean University, Northern Cyprus, \\ Famagusta, Turkey; e-mail: amir.teimourian@cc.emu.edu.tr
}

In this study, the effects of entrainment of a fluid through a perforated surface on suppression of the vortex street behind a perforated square cylinder have been studied experimentally. The wake region has been investigated in terms of coherent flow structure, time averaged properties and effectiveness of different perforations. The quantitative measurements revealed that the perforated surfaces are only effective within a width interval of $y / D= \pm 1.0$. It has been observed that in the near wake region up to approximately $1.5 \mathrm{D}$ downstream the wake, the shedding phenomenon has been suppressed significantly. It has been also demonstrated that velocity profiles and flow structure have been affected by different perforated surfaces and, as a result, coherent structures have been diminished considerably.

Keywords: vortex shedding, flow control, coherent flow structure, perforated square cylinder

\section{Nomenclature}

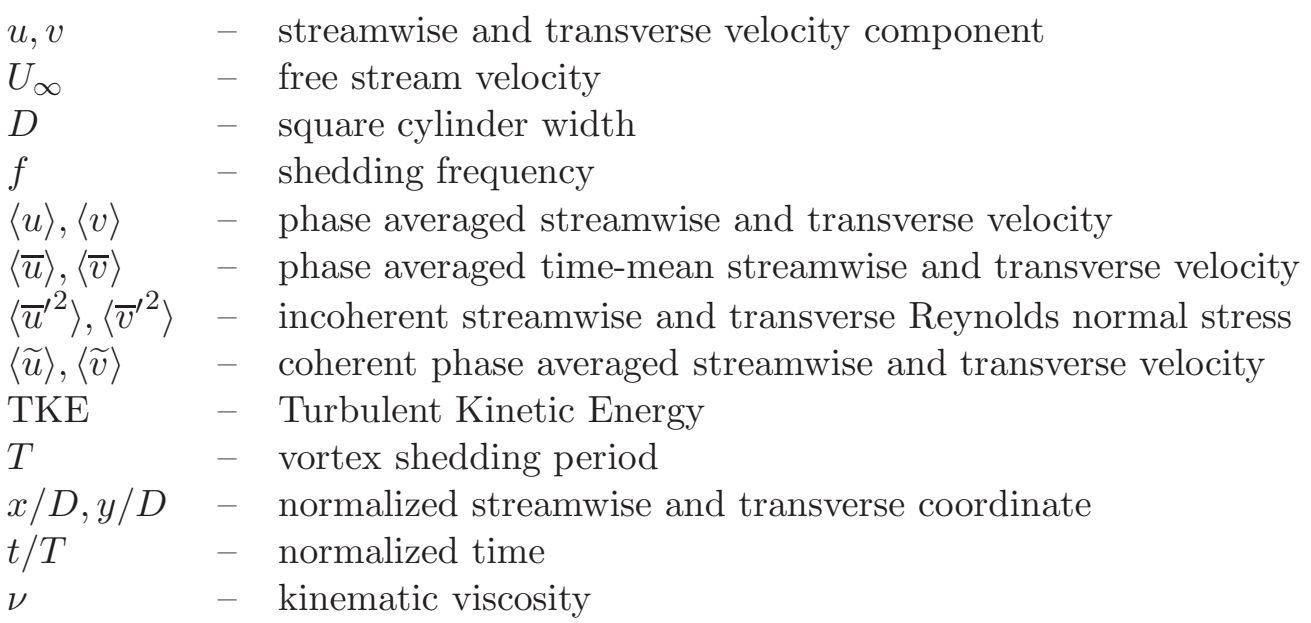

\section{Introduction}

The vortex shedding phenomenon from bluff bodies has been investigated on geometries and arrangements having different applications in civil engineering, wind engineering and aerospace engineering. The unsteady loading behavior which is induced by vortex shedding in the wake region requires great consideration and, as a result, has attracted many researchers. Such fluctuating forces are the main concern during design stages of industrial systems where the vortexinduced vibration can have an undesirable effect on the structure. In the context of a square cylinder, Okajima (1982), Saha et al. (2000), Saha (2013), Hacışevki and Teimourian (2015), Sohankar et al. (2015b) and many other researchers conducted extensive studies on the vortex shedding phenomenon. They investigated different features of vortex shedding behind square 
cylinders and reported wake flow structures, Strouhal number variation and other aerodynamic parameters.

The fluctuating forces are derived from the wake structure and, therefore, any endeavor for suppression of the vortex street to reduce such a destructive feature is valuable. For this purpose, various passive and active flow-control methods have been employed by engineers to protect the structures against the damaging fluid forces acting on the bluff bodies. Sakamoto et al. (1997), Alam et al. (2002), Malekzadeh and Sohankar (2012) and Igarashi (1997) investigated passive flow control by employing a control plate or a rod upstream a square cylinder as a means of controlling the vortex shedding. They all reported a considerable reduction on the mean and the fluctuating forces acting on the square cylinder. It was observed that while the drag force on the square cylinder was significantly reduced, the fluctuating lift was also suppressed.

On the other hand, Çuhadaroğlu et al. (2007), Çuhadaroğlu and Turan (2009), Çuhadaroglu (2009), Turhal and Çuhadaroğlu (2010) and Sohankar et al. (2015a) employed an active control method with injection of a fluid through the surface of the square cylinder to reduce the damaging effect of the vortex shedding phenomenon. Çuhadaroğlu et al. (2007) conducted an experimental study to investigate the injection effects on the pressure coefficient and drag coefficient of a perforated square cylinder at high Reynolds numbers between $R e=10000$ and 24000 . Different configurations of injection through the front, top and rear surfaces of the cylinder have been employed. The results revealed that injection through the rear face decreased the drag force. However, injection of the fluid through the front face demonstrated opposite results and caused an increase in drag force. Moreover, injection through other faces demonstrated negligible effects. Turhal and Çuhadaroğlu (2010) experimentally studied variation of the pressure coefficient, drag coefficient and Strouhal number of a perforated square cylinder (horizontal and diagonal) with having the fluid injected through various surfaces at high Reynolds numbers. The result revealed that in the case of a diagonal square cylinder, the surface injection through the top-rear, rear and all surfaces reduced the drag coefficient. However, only the injection through all surfaces of a horizontal square cylinder could result in a reduction of the drag coefficient. Numerical study on control of fluid flow of by injection through surfaces of a square cylinder, which were reported by Sohankar et al. (2015a), is one of the latest studies in this context. The simulation demonstrated that in the case of fluid injection through the front surface, the Strouhal number decreased by increasing the injection parameter, while aerodynamic force fluctuations were increased. On the other hand, the injection of fluid through the rear face caused a reduction in the Strouhal number and drag coefficient.

To sum up, the review shows that even though various passive and active flow control over a square cylinder have been investigated, there is still a gap in the literature. The previous studies tackled the problem of the perforated square cylinder together with active control theory. Although, the ultimate aim of these studies was the suppression of vortex shedding in the wake region of the square cylinder, most of the investigations reported variation of aerodynamic parameters such as the drag and pressure coefficient, and very limited results are available in terms of the wake flow structure and vortex street. Therefore, the objective of this study is to investigate the effects of perforating a square cylinder on the flow structure and suppression of vortex shedding. The suppression of vortex shedding has been studied by employing a triple decomposition technique rather than classical Reynolds decomposition to distinguish the incoherent turbulent flow fluctuation from the coherent vortex shedding structure for a better understanding of this phenomenon. The downstream wake behind the perforated square cylinder has been measured quantitatively by employing hotwire anemometry, and phase averaged properties have been presented. Moreover, coherent and incoherent structures of the downstream wake behind perforated square cylinders have been identified. The effectiveness of suppression of the vortex street in the wake region have been probed. 


\section{Experimental set-up and methodology}

The investigations have been conducted in an open-return subsonic wind tunnel with a $0.5 \mathrm{~m} \times 0.5 \mathrm{~m}$ test section and $1.0 \mathrm{~m}$ length available at Aerodynamic Laboratory of Eastern Mediterranean University. The wind tunnel speed was controlled by a Danfoss frequency controller with the maximum speed of $30 \mathrm{~m} / \mathrm{s}$. The experiment was conducted at a free stream velocity $U_{\infty}=10.5 \pm 2 \% \mathrm{~m} / \mathrm{s}$ with a measured turbulence intensity of $0.6 \%$ at this speed. A perforated hollow square cylinder with a cross section of $25 \mathrm{~mm} \times 25 \mathrm{~mm}$ with the corresponding blockage ratio of $5 \%$ was selected for the experiment.

The square cylinder model was constructed from aluminum, and four different perforations have been drilled with a CNC machine with accuracy $\pm 0.001 \mathrm{~mm}$. The experimental setup and schematic of the perforated square cylinders are illustrated in Figs. 1 and 2, respectively. Each face of the square cylinder was perforated with holes $\phi=2 \mathrm{~mm}$ in diameter uniformly distributed on the perforated surface. The Reynolds number of the experiment was $\operatorname{Re}=18500$ $\left(\operatorname{Re}=\left(U_{\infty} D\right) / \nu\right)$ based on the side-length of the square cross-section of the cylinder, which resulted in turbulent vortex shedding behind the square cylinder in the domain of interest $(0.5<x / D<4.0)$.

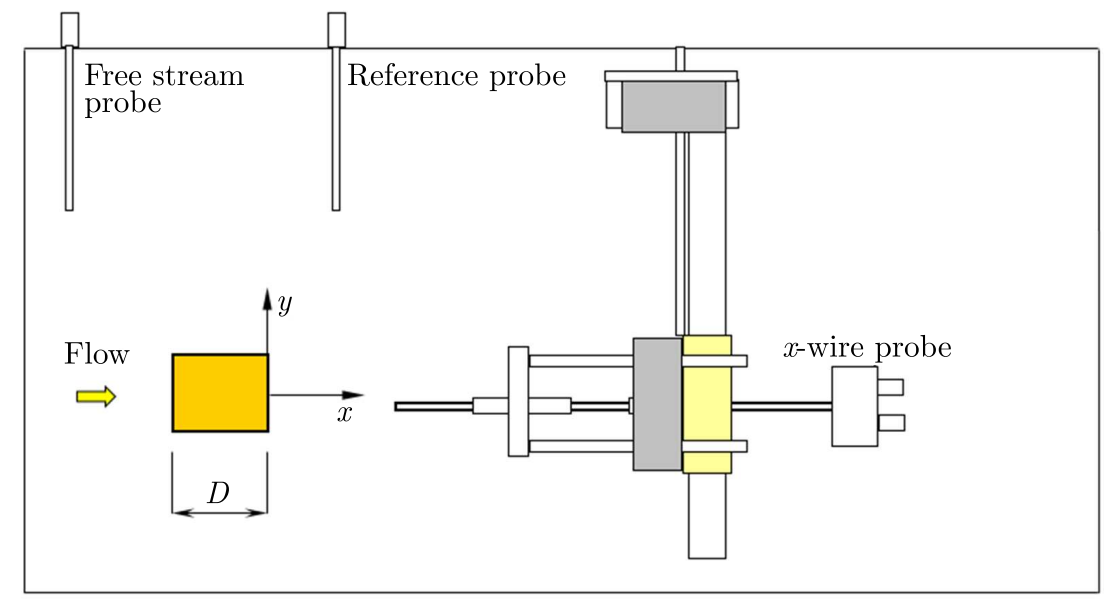

Fig. 1. Experimental setup and coordinates (top view)

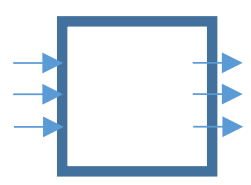

Front-rear (P0)

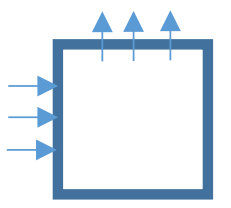

Front-one face (P1)

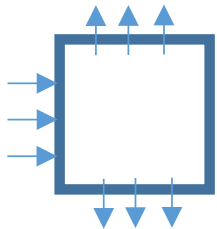

Front-two faces (P2)

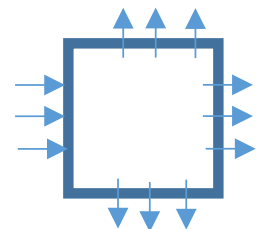

All faces (P3)

Fig. 2. Schematic of perforated square cylinders

Velocity field measurements have been acquired by employing TSI FlowPoint 1500 constant temperature anemometry hotwire system by means of two S types (1210-T1.5) and one X type (1240-T1.5) hotwires. Two velocity components ( $u$ and $v$ ) were acquired by means of an X hotwire probe, while free stream velocity and reference signal of periodic shedding were acquired by means of $\mathrm{S}$ hotwire probes. The $\mathrm{X}$ probe was mounted on a three-axis traverse mechanism to traverse in the $x y$ domain during data acquisition with an accuracy of $\pm 0.25 \mathrm{~mm}$. The velocity components for a given streamwise location $x / D$, were acquired at various lateral locations $y / D$ within \pm 2.6 . All the measurements were carried out at the midpoint of the test section in the $z$-direction. The acquired data was filtered by considering the frequency of shedding with the 
reference waveform by implementing MATLAB/Simulink to produce the characteristic vortex shedding waveform. Finally, the filtered data was analyzed by implementing the triple decomposition and ensemble averaging method (Hussain, 1986; Reynolds and Hussain, 1972) to produce the phase averaged properties. This technique provides a better understanding to distinguish the coherent structure and incoherent turbulent fluctuations. The instantaneous velocity $u$, can be decomposed into time-mean averaged component $\bar{u}$, coherent component $\widetilde{u}$ and incoherent component (turbulent fluctuations) $u^{\prime}$

$$
u=\bar{u}+\widetilde{u}+u^{\prime} \quad v=\bar{v}+\widetilde{v}+v^{\prime}
$$

The purpose is to obtain the dependence of these components on time (normalized with respect to total period $T$ ) during one complete cycle of vortex shed. A reference signal (the periodic vortex shedding velocity fluctuation in the present study) has been selected and the velocity signals ( $u$ and $v$ component) at the selected domain point have been acquired. The phase averages are then computed by sorting the instantaneous velocity components into 60 different bins of different phases obtained by equally subdividing the intervals between peaks of the output of the phase detector probe. Then, corresponding to same $t / T$ of consecutive reference cycles, the acquired velocity components at the same instant are summed and averaged. This process is repeated for different times during one cycle to construct the variation of properties $\langle u\rangle,\langle v\rangle$, $\langle\bar{u}\rangle,\langle\bar{v}\rangle,\left\langle\bar{u}^{\prime 2}\right\rangle,\left\langle\bar{v}^{\prime 2}\right\rangle,\langle\widetilde{u}\rangle,\langle\widetilde{v}\rangle$ and TKE $\left(\left\langle\widetilde{u}^{2}\right\rangle+\left\langle\widetilde{v}^{2}\right\rangle\right)$ across one complete cycle of vortex shedding. A modified Navier-Stokes equation was obtained by substituting the triple decomposition of the $u$ and $v$ components of velocity and by applying phase averaging techniques as shown below

$$
\langle u\rangle=\frac{1}{n} \sum_{1}^{n} u \quad\langle\widetilde{u}\rangle=\frac{1}{n} \sum_{1}^{n} \widetilde{u} \quad\left\langle u^{\prime}\right\rangle=\frac{1}{n} \sum_{1}^{n} u_{i}^{\prime}
$$

where $n$ is the number of cycles used for phase averaging and $\widetilde{u}$ is the value of coherent component and $u^{\prime}$ is the value of incoherent component at the same normalized time successive cycles

$$
\langle\bar{u}\rangle=\frac{1}{m} \sum_{1}^{m}\langle u\rangle \quad\langle\overline{\widetilde{u}}\rangle=\frac{1}{m} \sum_{1}^{m}\langle\widetilde{u}\rangle \quad\left\langle\overline{u^{\prime}}\right\rangle=\frac{1}{m} \sum_{1}^{m}\left\langle u^{\prime}\right\rangle
$$

where $m$ is the number of phase averages used in one cycle to construct phase averages of the successive cycles. Such a definition can be applied for any velocity components or product of velocity components. Consequently, applying the phase averaging concept to the Navier-Stokes momentum equation will result in the modified Navier-Stokes equation. Furthermore, the coherent Turbulence Kinetic Energy (TKE) can be defined as the following equation

$$
\mathrm{TKE}=\frac{1}{2} \overline{\widetilde{u}_{i} \widetilde{u}_{i}}=\frac{1}{2}\left(\overline{\widetilde{u}_{1} \widetilde{u}_{1}+\widetilde{u}_{2} \widetilde{u}_{2}}\right)=\frac{1}{2}(\overline{\widetilde{u} \widetilde{u}+\widetilde{v} \widetilde{v}})
$$

\section{Spectral analysis}

In order to identify the vortex shedding frequency and quantitative comparison between different perforated square cylinders, Fast Fourier Transform (FFT) has been implemented on the acquired velocity data. Therefore, the dominant shedding frequency $f$ can be observed as a single peak corresponding to the Strouhal number, St $=f D / U_{\infty}$, in the wake behind the cylinder. The instantaneous velocity has been acquired at various $x / D$ downstream the wake and various transverse directions have been used for frequency spectra determination. The dominant shedding frequency has been found as $f=43.9 \mathrm{~Hz}$ that gives the Strouhal number St $=0.104$, identical to the corresponding Strouhal number of the non-perforated square cylinder. These dominant shedding frequencies have been acquired at positions outside the effective wake width of 
the perforated surface. Similarly, the shedding frequencies obtained by Turhal and Çuhadaroğlu (2010) at a similar position for the perforated square cylinder with injection demonstrated an insignificant alternation in the shedding frequency. However, the measurement inside the effective width of perforation were influenced by entrainment of the fluid through the perforated surfaces. It would be impossible to present all the power spectra due to space limitation. Therefore, for the sake of brevity, only a comparison between different perforated square cylinders and detailed investigation on perforated square cylinder P3 have been presented in Figs. 3 and 4, respectively.

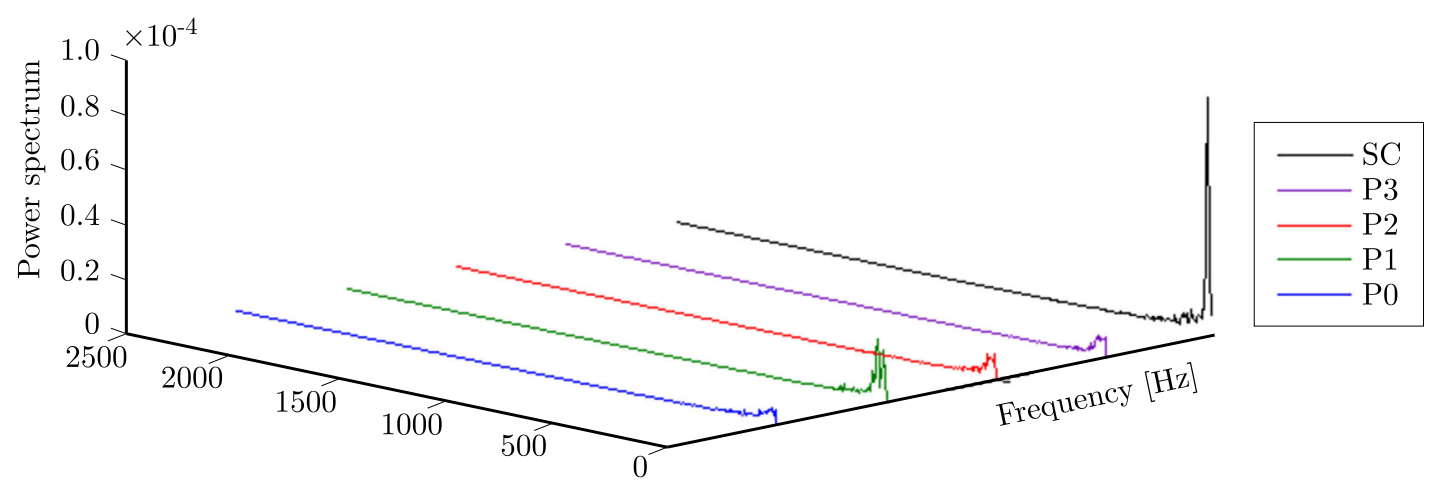

Fig. 3. Comparison of PSD of total transverse velocity fluctuations at $x / D=0.5$ and $y / D=0$ for perforated square cylinders P0, P1, P2, P3 and non-perforated square cylinders (SC)

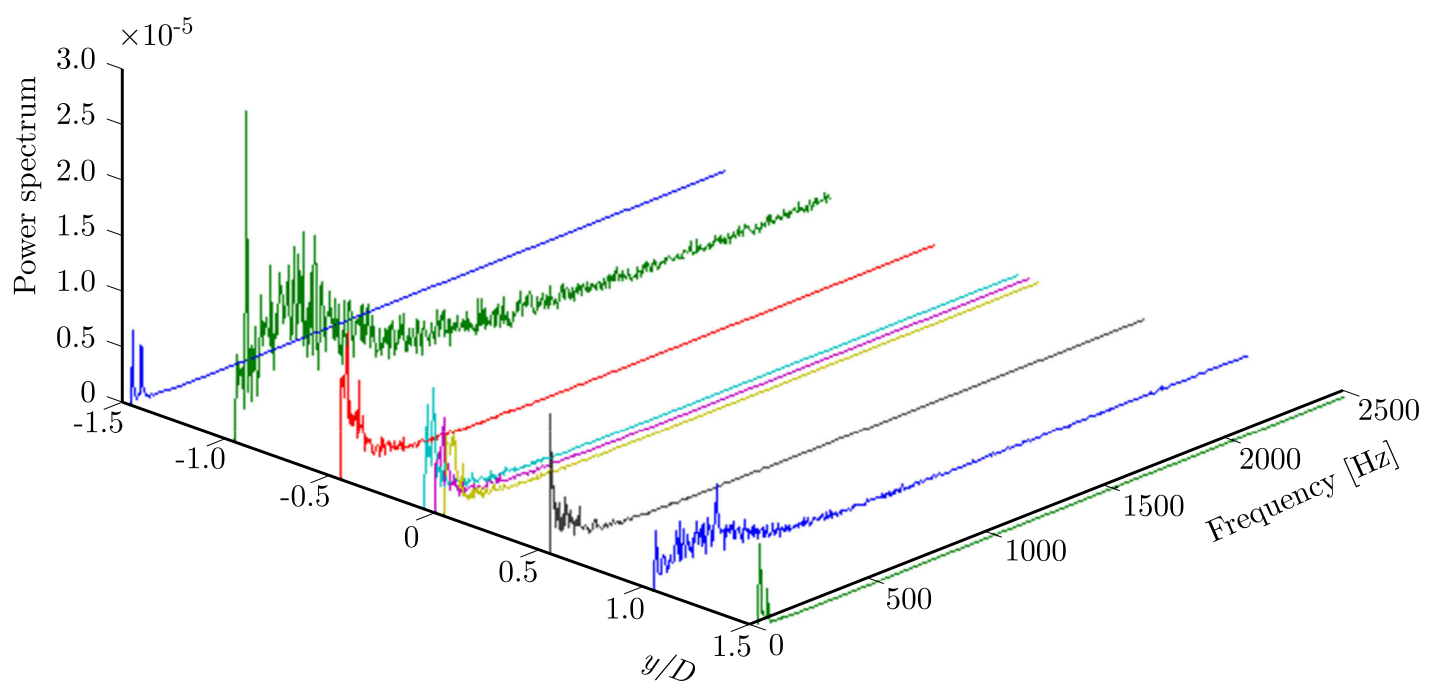

Fig. 4. PSD of perforated square cylinder P3 at $x / D=0.5$ for various transverse locations

Power Spectrum Density (PSD) analysis of total (i.e. random and coherent) transverse velocity fluctuations for different perforated square cylinders (P0, P1, P2 and P3) and non-perforated square cylinder (SC) measured at $x / D=0.5$ and $y / D=0$ (along centerline) in the downstream wake has been demonstrated in Fig. 3. As it can be seen from the figure, the frequency spectrum is clearly evident as a single strong peak in the wake of the non-perforated square cylinder. By contrast, in the wake region behind the perforated square cylinder, no dominant shedding frequency along the centerline is evident. Such an observation implied that the vortex shedding has been partially suppressed by fluid entrainment through the perforated surfaces.

Further investigations of PSD of total transvers velocity fluctuations acquired within the transverse direction interval $y / D= \pm 1.5$ for perforated square cylinder P3 have been illustrated in Fig. 4. It can be seen that the dominant shedding frequency is clearly evident for the wake region outside the interval $y / D= \pm 0.5$. However, inside this interval, where the perforated 
surfaces are effective, the power spectrum demonstrated evidence of no-single dominant peak with multiple peaks in the spectra. These multiple peaks indicated formation of the secondary vortex and suppression of the primary shedding due to entrainment of the fluid through the perforated surfaces.

\section{Phase averaged properties}

The objective of this study is to investigate the formation of the vortex shedding phenomenon in the wake region behind the perforated square cylinders. Development of the vortex street in the wake region behind perforated square cylinder P0 has been demonstrated in Fig. 5. The figure illustrates the variation of phase averaged streamwise velocity contours $\langle u\rangle$ versus normalized time $t / T$ during one complete cycle of oscillation for various $x / D$ downstream the wake region.

(a)

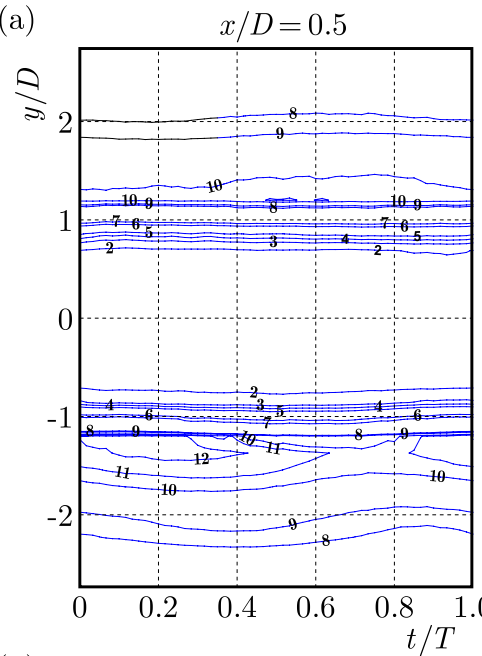

(b)

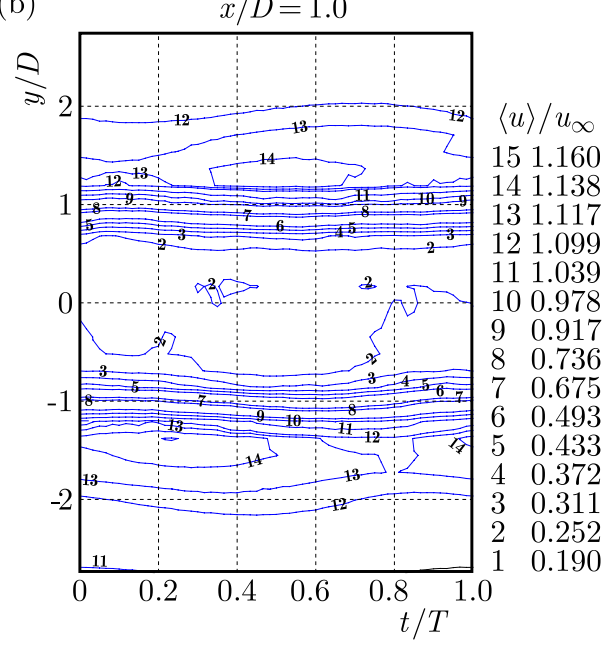<smiles>[14CH3]</smiles>
$\begin{array}{ll}18 & 1.250 \\ 17 & 1.207\end{array}$ 161.179 151.173 $\begin{array}{ll}14 & 1.169 \\ 13 & 1.161\end{array}$

$\begin{array}{ll}12 & 1.145 \\ 11 & 1.128\end{array}$

111.128

$9 \begin{array}{ll}9 & 1.083 \\ 9 & 1.069\end{array}$

$8 \quad 1.069$

$\begin{array}{lll}7 & 0.822\end{array}$

$\begin{array}{ll}6 & 0.751 \\ 5 & 0.537\end{array}$

$4 \quad 0.465$

$3 \quad 0.394$

$\begin{array}{ll}2 & 0.263 \\ 1 & 0.180\end{array}$ 0.180 (c)

c)

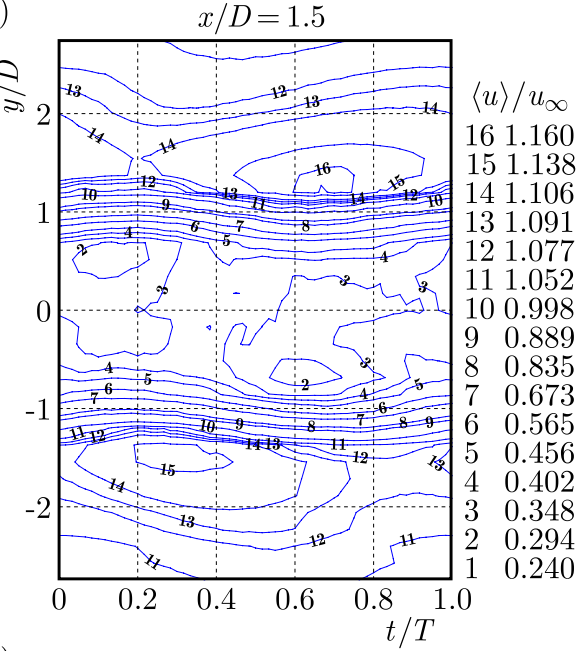

(d)

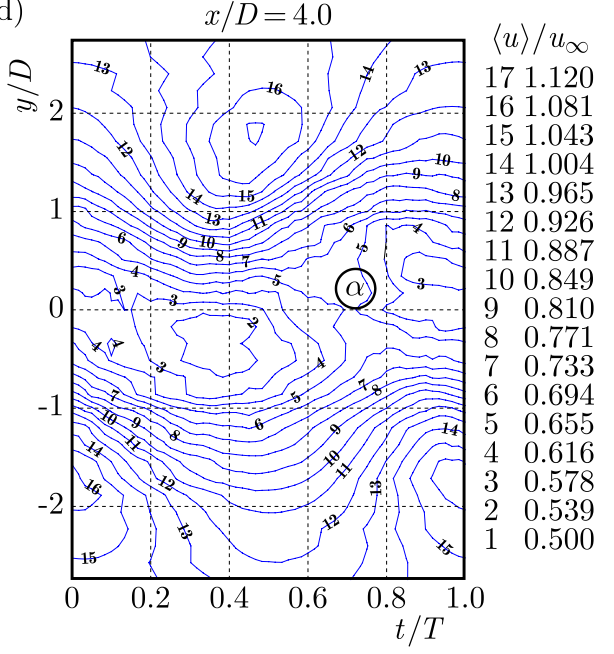

Fig. 5. Comparison of phase averaged streamwise velocity $\langle u\rangle$ for perforated square cylinder P0 acquired at: (a) $x / D=0.5$, (b) $x / D=1.0$, (c) $x / D=1.5$, (d) $x / D=4.0$

It can be seen from the figure that the vortex shedding has been completely suppressed by the entrainment of the fluid into the wake region through the perforated surfaces in the wake region up to approximately $x / D=1.5$. In the near wake region of the perforated square cylinder, i.e. $x / D=0.5$, while within the interval $y / D= \pm 1$ there is no evidence of vortex shedding, some vortices are being rolled beyond the edges of the square cylinder. As the probe moves downstream the wake, the vortex shedding process is being developed and at $x / D=1.5$, the $\langle u\rangle$ 
contours exhibit patterns corresponding to vortices being shed periodically. In addition to two peaks beyond the edges of the perforated square cylinder, two low level zone phase drifted by half the period corresponding to edges of the perforated square cylinder are also evident. Eventually, the streamwise phase average velocity contours at $x / D=4.0$, where the perforated surfaces are no more effective, demonstrate classical vortex shedding. Through such development, the peaks drift away from the centerline. In contrast, the two low level zones are shifted towards the centerline behind the perforated square cylinder. Such a flow pattern is comparable to the streamwise velocity in the wake region of the square cylinder as reported by Hacişevki and Teimourian (2015).

Figure 6 illustrates the phase averaged transverse velocity component contours at two different distances downstream the wake region. Similarly to the streamwise velocity component, the peaks occur during a half of the period phase $(t / T=0.5)$. However, comparison between streamwise and transverse velocity component measured at $x / D=4.0$ reveals that the transverse velocity peaks (point $\alpha$ as illustrated in Fig. 6) lag behind the streamwise velocity peaks with a phase shift equal to $1 / 4$ of the cycle. Therefore, the point $\alpha$ as illustrated in Fig. 5, corresponds to normalized time at which the transverse velocity reaches the peak value in Fig. 6 . The contours around this peak region imply a remarkable lateral momentum transfer towards the growing vortex on the other edge of the square cylinder. However, such a phenomenon has been suppressed by the perforated surface near the wake, i.e. $x / D=1.0$, where the perforated surfaces are effective.

(a)

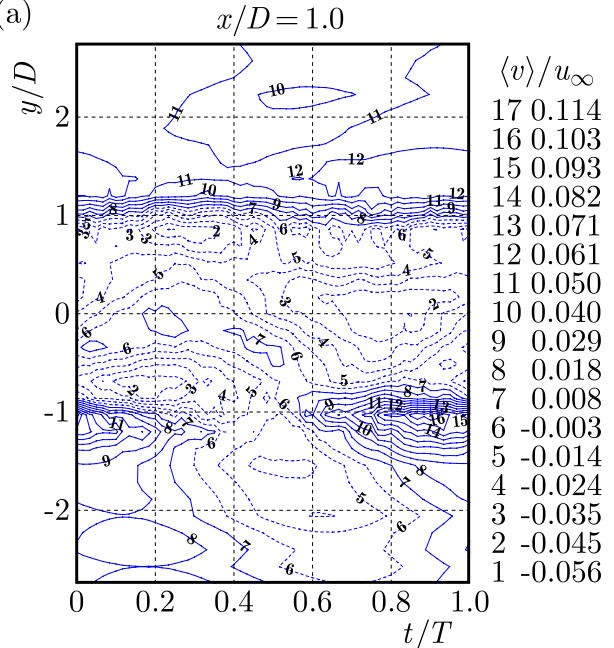

(b)

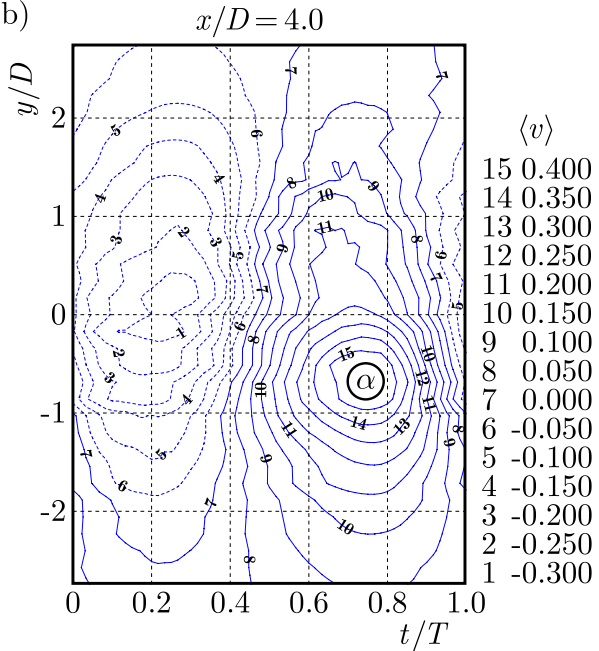

Fig. 6. Comparison of the phase averaged transverse velocity $\langle v\rangle$ for perforated square cylinder P0 acquired at (a) $x / D=1.0$ and (b) $x / D=4.0$

Furthermore, a comparison between the phase averaged streamwise velocities in the wake region of the non-perforated square cylinder and four different perforated square cylinders measured at $x / D=1.5$ have been presented in Fig. 7. From this figure it can be observed that while in the wake region of the non-perforated square cylinder the rolling vortices are clearly apparent with periodic flow patterns, the development of the vortex shedding phenomenon in the wake region behind perforated square cylinders have been delayed. Moreover, it can be observed that perforated square cylinders P0 and P3 delayed such development more effectively as a result of entraining the fluid particle directly into the wake region by employing the perforated rear face.

As discussed previously, triple decomposition would provide a better illustration of the vortex shedding phenomenon. Therefore, to investigate the development of a coherent structure of the shedding phenomenon, coherent Turbulent Kinetic Energy production in the wake region behind perforated square cylinder P1 has been depicted in Fig. 8. 
(a)

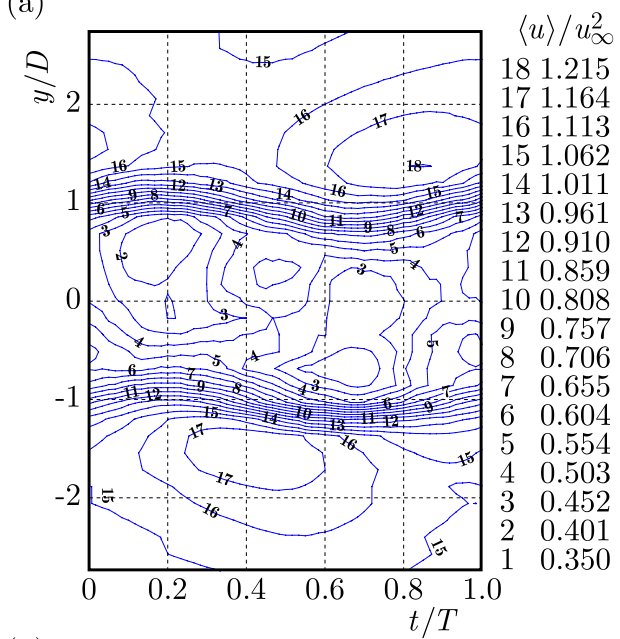

(b)

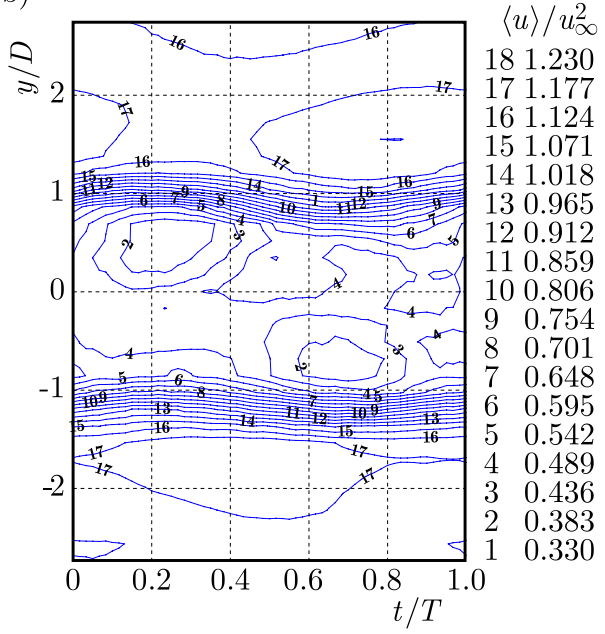

(c)

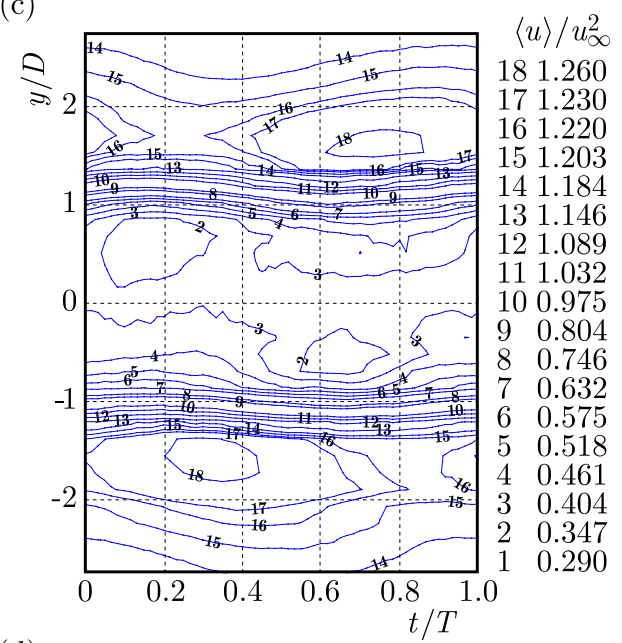

(d)

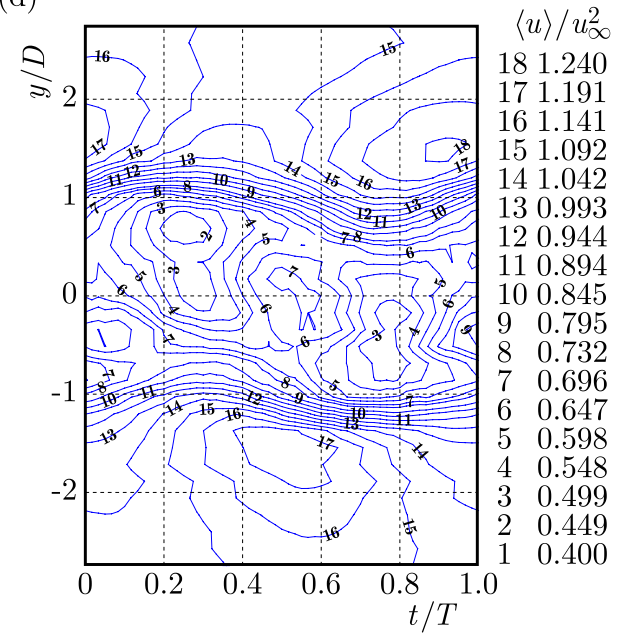

Fig. 7. Comparison of phase averaged streamwise velocity $\langle u\rangle$ acquired at $x / D=1.5$ for perforated square cylinder: (a) P1, (b) P2, (c) P3 and (d) non-perforated square cylinder (SC)

(a) $\quad x / D=0.5$

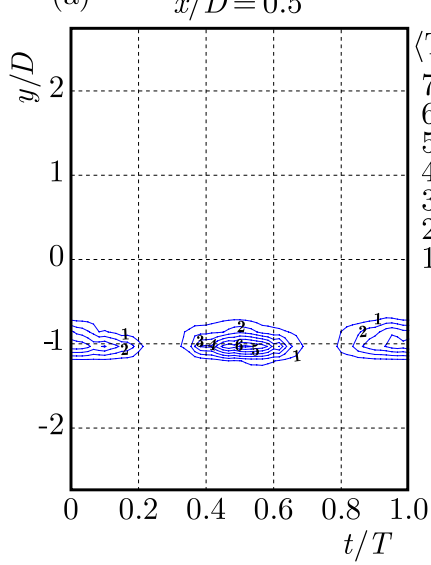

(b)

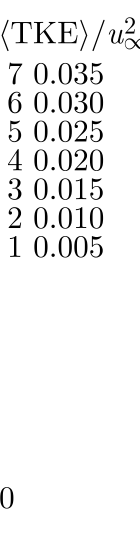

$x / D=1.0$

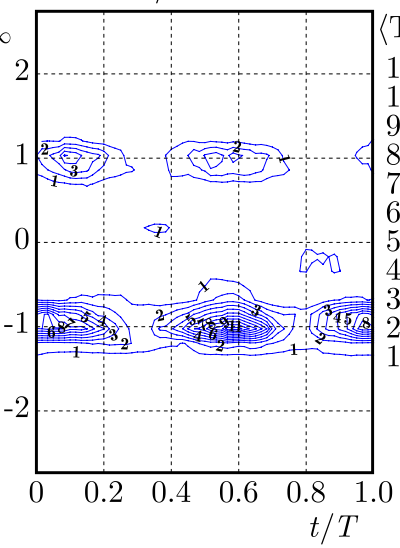

(c) $\quad x / D=1.5$

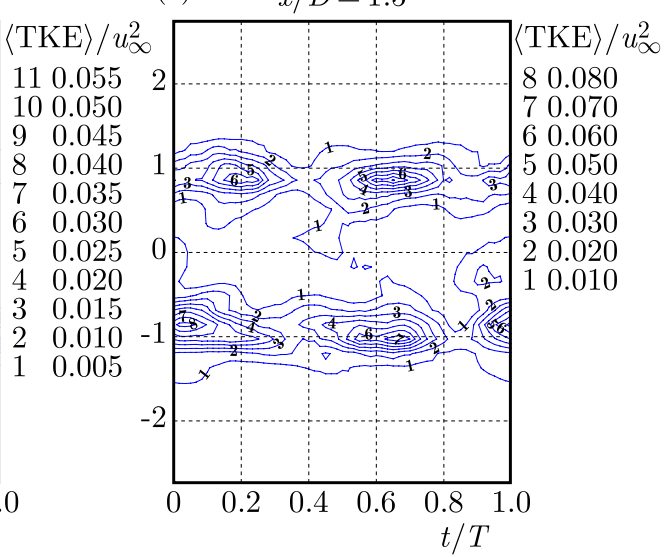

Fig. 8. Coherent Turbulent Kinetic Energy production in the wake region of perforated square cylinder P1 acquired at: (a) $x / D=0.5$, (b) $x / D=1.0$, (c) $x / D=1.5$ 
It can be seen from the figure that near the wake region while coherent structures have been formed beyond the right edge, the wake of the left edge shows no evidence of the coherent structure. As the probe moved downstream, the perforated surface is losing its effectiveness and, as a result, the first evidence of the coherent structure on the left edge has been also developed. Farther downstream the wake (i.e. $x / D=4.0$ ), the two coherent structures are combined and form a larger structure as wide as the wake.

The relative reduction in coherent TKE production with respect to the non-perforated cylinder can be a measure of effectiveness of such perforation. As a result, the relative reduction in coherent TKE peak production for P1 (compared with the non-perforated square cylinder) varies form $66 \%$ to $27 \%$ between $x / D=0.5$ to 1.5 downstream the wake, and beyond $x / D=1.5$ the effectiveness drops significantly to approximately $7 \%$ at $x / D=3.0$. The effectiveness of other perforated surfaces for coherent streamwise and transverse velocities and coherent TKE have been illustrated in Table 1.

Table 1. Relative reduction in the coherent structure peak value and TKE with respect to the non-perforated cylinder

\begin{tabular}{c|c|c|c|c|c|c|c|c|c|}
\cline { 2 - 11 } \multicolumn{1}{c|}{} & \multicolumn{3}{c|}{$x / D=0.5$} & \multicolumn{3}{c|}{$x / D=1.0$} & \multicolumn{3}{c|}{$x / D=1.5$} \\
\cline { 2 - 11 } & $\langle\widetilde{u}\rangle$ & $\langle\widetilde{v}\rangle$ & TKE & $\langle\widetilde{u}\rangle$ & $\langle\widetilde{v}\rangle$ & TKE & $\langle\widetilde{u}\rangle$ & $\langle\widetilde{v}\rangle$ & TKE \\
\hline \hline P0 & 65 & 86 & 90 & 52 & 73 & 88 & 36 & 56 & 64 \\
\hline P1 & 42 & 64 & 67 & 24 & 54 & 38 & 7 & 16 & 27 \\
\hline P2 & 69 & 86 & 92 & 52 & 73 & 88 & 21 & 50 & 55 \\
\hline P3 & 81 & 86 & 96 & 64 & 77 & 88 & 39 & 63 & 73 \\
\hline
\end{tabular}

\section{Time averaged properties}

Better understanding of the flow structure also requires knowledge how to quantify development and decay of flow properties such as streamwise velocity, transverse velocity and turbulent stresses downstream the wake region. In this Section, a quantitative comparison of the phase averaged time-mean streamwise velocity and the phase averaged time-mean turbulent stresses measured at various $x / D$ in the wake region have been presented. Phase averaged time-mean properties such as streamwise and transverse velocities are normalized with the free stream velocity $U_{\infty}$, and the turbulent normal and shear stress are normalized with $U_{\infty}^{2}$.

The variation of phase averaged streamwise velocity contours $\langle u\rangle$ versus normalized time $t$ during one complete cycle of oscillation have been presented in the previous Section. At a given $y / D$, averaging with respect to $t / T$ in one cycle yields the phase averaged time-mean $\langle\bar{u}\rangle$ or, traditionally known, the time mean $\bar{u}$ of the streamwise velocity. For the streamwise velocity, this two averaging techniques result in the same values as both imply the same averaging. The phase averaged time-mean can be obtained by averaging across one cycle with the values obtained across all acquired cycles, while the time mean is computed across all cycles in the direct time averaging process. Variation of the phase averaged time-mean streamwise velocity $\langle\bar{u}\rangle$ against the lateral position $y / D$ for different perforated square cylinders is demonstrated in Fig. 9. In thise figure, symmetrical variation of $\langle\bar{u}\rangle$ profile with respect to the wake axis is clearly apparent. It can be observed that for the case of perforated square cylinder P1 with no perforated surface in the rear face, the $u$ profile measured near the wake $(x / D=0.5)$ decreases up to the centerline and then increases gradually. However, in case of $\mathrm{P} 0$ with a perforated rear face, the $u$ profile remains approximately constant with some fluctuation corresponding to the measurement exactly behind the perforations. Moreover, it can be also observed that further downstream the wake, the effect of perforated surfaces decays considerably. 
(a)

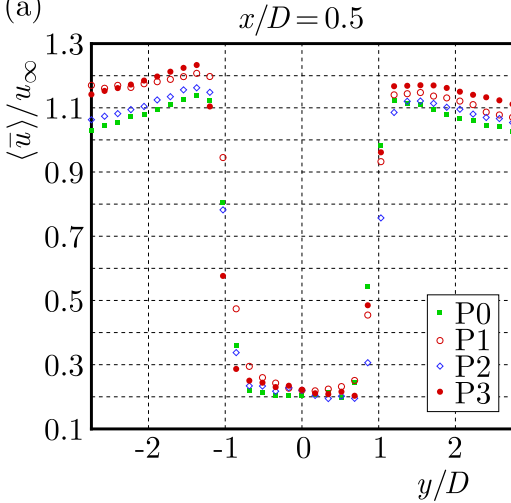

(b)

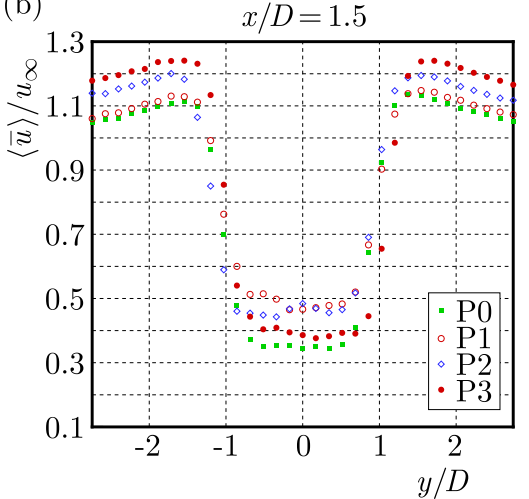

(c)

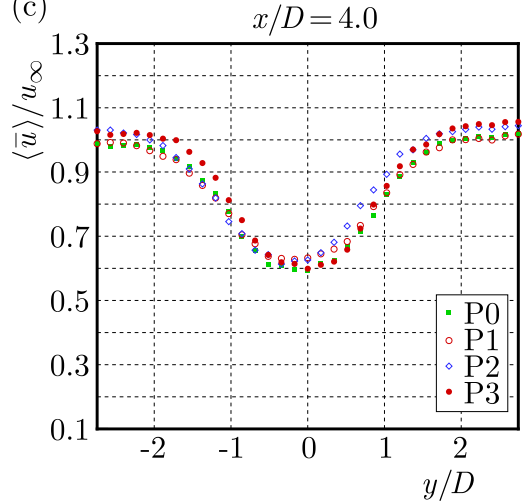

Fig. 9. Comparison of variation of $\langle\bar{u}\rangle$ for different perforated square cylinders acquired at: (a) $x / D=0.5$, (b) $x / D=1.5$, (c) $x / D=4.0$

The phase averaged time-mean incoherent streamwise and transverse stresses downstream the wake for different perforated square cylinders measured at $x / D=1.0$ and 4.0 are illustrated in Fig. 10. In the figure, it can be observed that both streamwise and transverse normal stresses significantly decay compared with the non-perforated square cylinder (SC) in the interval of $y / D= \pm 1.0$ near the wake. However, further downstream the wake at $x / D=4.0$, incoherent stresses reach a value close to the non-perforated square cylinder. It can be observed that P0 exhibits the lowest streamwise and transverse normal stress. Such an observation is as a result of direct entrainment of the fluid through the perforated rear side into the wake region together with having the highest entrainment flow rate. Moreover, in the near wake region $(x / D=1.0)$ both streamwise and transverse stresses exhibit double peaks corresponding to the vortex shed from the edges. However, such a profile transforms gradually to a single peak profile as the vortices migrate toward the centerline.

(a)
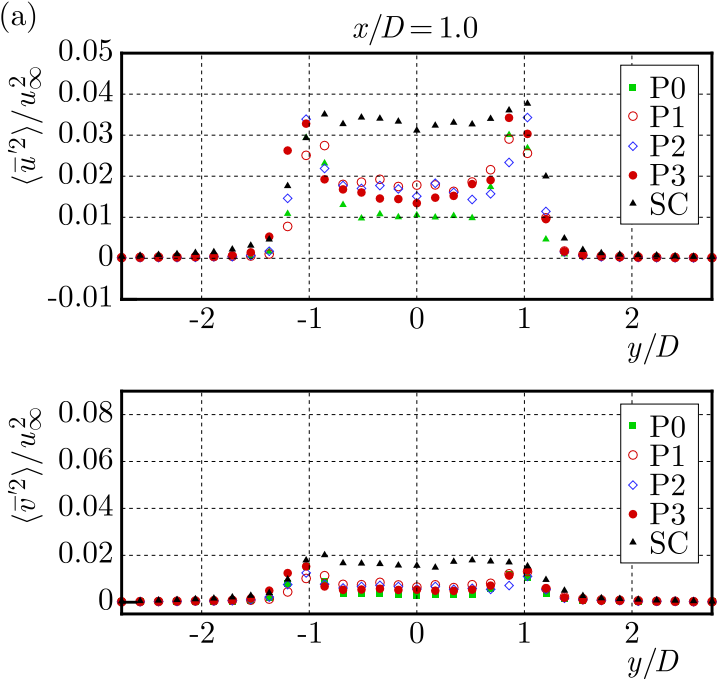

(b)
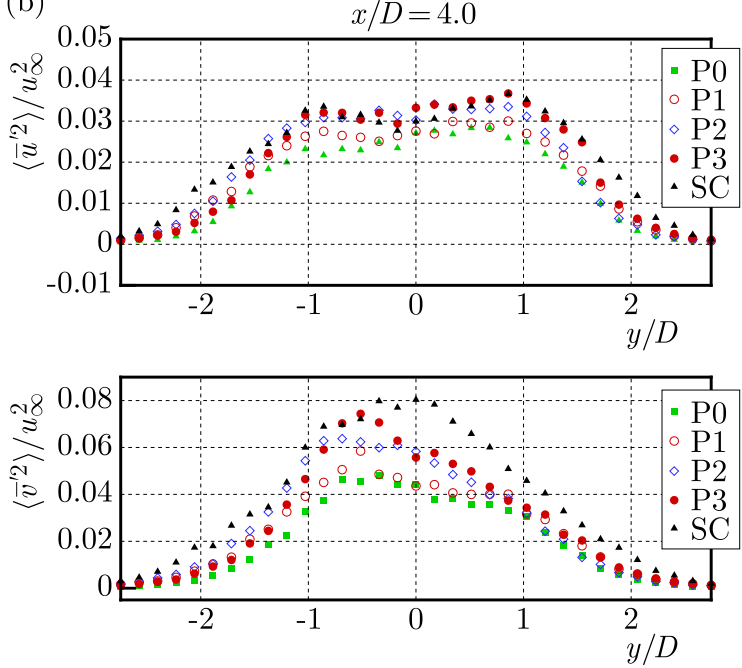

Fig. 10. Comparison of the phase averaged time mean incoherent normal Reynolds stress for different perforations acquired at: (a) $x / D=1.0$, (b) $x / D=4.0$

Clearly, an insufficient number of phases during one cycle resulted in discrete variation for phase averaging rather than a continuous variation of the properties. However, in this study with employing 60 phases during one cycle, the time mean phase averaged properties over one cycle are zero within an accuracy of $\pm 1 \%$. Such an observation has been illustrated in Fig. 11 for coherent streamwise and transverse velocity components. 

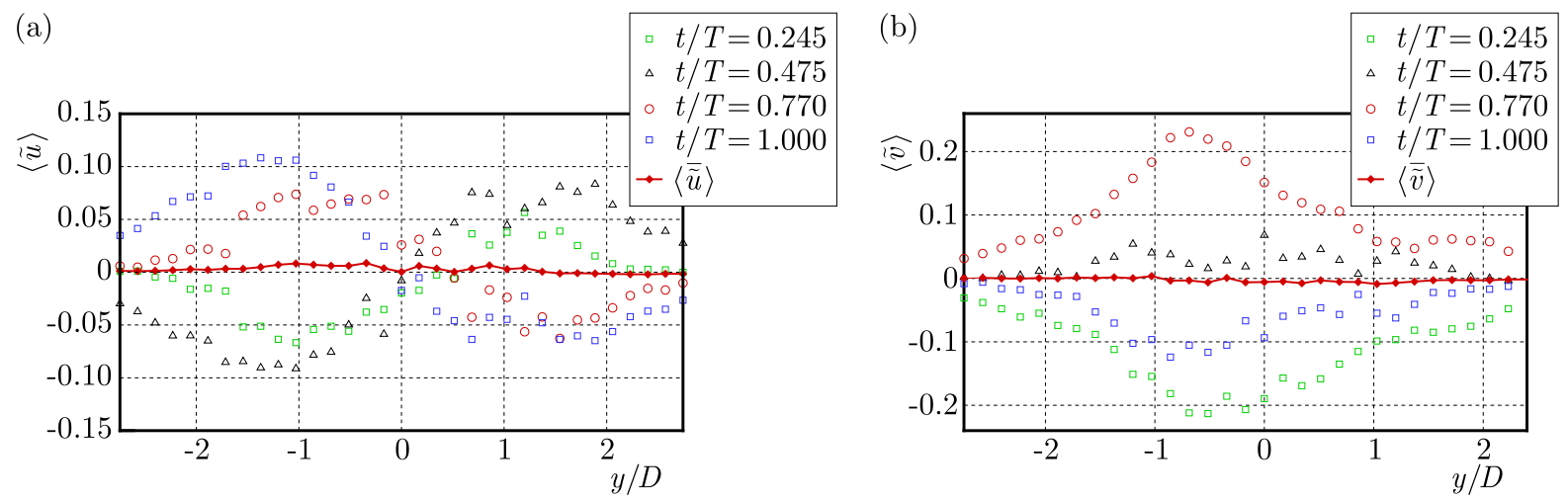

Fig. 11. Phase averaged coherent velocity components profile measured at $x / D=4.0$ at various normalized times

\section{Conclusion}

In this study, the effect of entrainment of a fluid through perforated surfaces on suppression of the vortex street behind a perforated square cylinder has been studied experimentally. To probe the vortex formation process, different characteristics and coherent structures in the downstream wake have been analyzed. It has been observed that the perforated surfaces are effective only within the effective width $y / D= \pm 1.0$, and up to $1.5 D$ in the downstream wake. Although previous studies reported that high injection through the rear surface is required to decrease the vortex shedding frequency. It has been found that even for a low entrainment flow rate, the shedding phenomenon has been affected significantly. Thus, observation of multiple peaks in the spectra indicates the secondary vortex formation and suppression of the primary shedding due to entrainment of the fluid through the perforated surfaces. The result shows that different perforated surface front-rear, front-one face, front-two faces and all faces have some effects on the velocity profiles, flow structure and vortex street. Asymmetric coherent flow structures behind the perforated square cylinder have been observed and the effectiveness of this perforation has been presented in terms of reduction in the coherent structure peak value. It has been revealed that the square cylinder with all faces perforated, has given the most significant relative reduction in the coherent TKE production compared with other perforations.

\section{References}

1. Alam M.M., Moriya M., Takai K., Sakamoto H., 2002, Suppression of fluid forces acting on two square prisms in a tandem arrangement by passive control of flow, Journal of Fluids and Structures, 16, 1073-1092

2. ÇuhadaroĞLu B., 2009, A numerical study on turbulent flow around a square cylinder with uniform injection or suction, International Journal of Numerical Methods for Heat and Fluid Flow, 19, 708-727

3. ÇuhadaroĞlu B., Akansu Y.E., Turhal A.Ö., 2007, An experimental study on the effects of uniform injection through one perforated surface of a square cylinder on some aerodynamic parameters, Experimental Thermal and Fluid Science, 31, 909-915

4. ÇuhadaroĞLu B., Turan O., 2009, Numerical simulation of turbulent flow around a square cylinder with uniform injection or suction and heat transfer, Numerical Heat Transfer, Part A: Applications, 55, 163-184

5. Hacişevki H., Teimourian A., 2015, Comparison of flow structures in the wake region of two similar normal flat plates in tandem and a square cylinder, Experimental Thermal and Fluid Science, 69, 169-177 
6. Hussain A., 1986, Coherent structures and turbulence, Journal of Fluid Mechanics, 173, 303-356

7. IGARASHI T., 1997, Drag reduction of a square prism by flow control using a small rod, Journal of Wind Engineering and Industrial Aerodynamics, 69, 141-153

8. Malekzadeh S., Sohankar A., 2012, Reduction of fluid forces and heat transfer on a square cylinder in a laminar flow regime using a control plate, International Journal of Heat and Fluid Flow, 34, 15-27

9. Okалыма A., 1982, Strouhal numbers of rectangular cylinders, Journal of Fluid Mechanics, 123, 379-398

10. Reynolds W., Hussain A., 1972, The mechanics of an organized wave in turbulent shear flow. Part 3. Theoretical models and comparisons with experiments, Journal of Fluid Mechanics, 54, $263-288$

11. SaнA A.K., 2013, Unsteady flow past a finite square cylinder mounted on a wall at low Reynolds number, Computers and Fluids, 88, 599-615

12. Saha A., Muralidhar K., Biswas G., 2000, Experimental study of flow past a square cylinder at high Reynolds numbers, Experiments in Fluids, 29, 553-563

13. Sakamoto H., Tan K., Takeuchi N., Haniu H., Suppression of fluid forces acting on a square prism by passive control, Journal of Fluids Engineering, 119, 506-511

14. Sohankar A., Khodadadi M., Rangraz E., 2015a, Control of fluid flow and heat transfer around a square cylinder by uniform suction and blowing at low Reynolds numbers, Computers and Fluids, 109, 155-167

15. Sohankar A., Mohagheghian S., Dehghan A., Manshadi M.D., 2015b, A smoke visualization study of the flow over a square cylinder at incidence and tandem square cylinders, Journal of Visualization, 18, 687-703

16. Turhal A.Ö., ÇuhadaroĞLu B., 2010, The effects of surface injection through a perforated square cylinder on some aerodynamic parameters, Experimental Thermal and Fluid Science, 34, $725-735$ 